\title{
Preparation and Drug-Release Kinetics of Porous Poly(L-lactic acid)/Rifampicin Blend Particles
}

\author{
Takashi Sasaki, Hiroaki Matsuura, and Kazuki Tanaka \\ Department of Materials Science and Engineering, University of Fukui, 3-9-1 Bunkyo, Fukui 910 8507, Japan \\ Correspondence should be addressed to Takashi Sasaki; sasaki@matse.u-fukui.ac.jp
}

Received 29 November 2013; Accepted 18 January 2014; Published 23 February 2014

Academic Editors: C. Bernal, A. Granville, D. Pavel, J. Puiggali, A. V. Raghu, A. Uygun, and A. Varesano

Copyright (C) 2014 Takashi Sasaki et al. This is an open access article distributed under the Creative Commons Attribution License, which permits unrestricted use, distribution, and reproduction in any medium, provided the original work is properly cited.

\begin{abstract}
Porous polymer spheres are promising materials as carriers for controlled drug release. As a new drug-carrier material, blend particles composed of poly(L-lactic acid) (PLLA) and rifampicin were developed using the freeze-drying technique. The blend particles exhibit high porosity with a specific surface area of $10-40 \mathrm{~m}^{2} \mathrm{~g}^{-1}$. Both the size and porosity of the particles depend on the concentration of the original solution and on the method of freezing. With respect to the latter, we used the drop method (pouring the original solution dropwise into liquid nitrogen) and the spray method (freezing a mist of the original solution). The release kinetics of rifampicin from the blend particles into water depends significantly on the morphology of the blend particles. The results show that the release rate can be controlled to a great extent by tuning the size and porosity of the blend particles, both of which are varied by parameters such as the solution concentration and the method of freezing.
\end{abstract}

\section{Introduction}

Recently, microspheres and capsules for controlled drug release have been developed by using biodegradable and biocompatible polymers [1-3]. Highly porous polymeric spheres are often used as drug carriers because their high porosity allows for a high efficiency of drug loading and facilitates controllable release [4-7]. As carrier materials, poly(Llactic acid) (PLLA), a typical biodegradable polymer, and its copolymer poly(lactic-co-glycolic acid) have been frequently studied [8-13]. Porous polymer spheres can be prepared using various techniques, among which freeze-drying from a dilute solution has been demonstrated to be a particularly excellent method $[14,15]$. We have investigated the properties of highly porous poly(L-lactic acid) (PLLA) materials prepared using the freeze-drying technique (FDPLLA), which exhibit very high specific surface areas of $10-40 \mathrm{~m}^{2} \mathrm{~g}^{-1}$, and have found that they crystallize at a lower temperature and at a higher crystallization rate than bulk amorphous PLLA $[15,16]$.

The capability for controlled drug release from FDPLLA has been demonstrated using bovine serum albumin (BSA) as a model drug compound [11]. The immersion of FDPLLA in a BSA aqueous solution results in BSA-loaded FDPLLA via a simple adsorption mechanism, which shows an extremely high efficiency of loading (up to $79 \mathrm{wt} \%$ with respect to PLLA). It was also observed that the release kinetics strongly depends on the porosity of FDPLLA, which can be controlled by the concentration of the original solution and the rate of freezing. Such a simple adsorption method allows us to obtain particles containing a large amount of the drug. However, the adsorption tends to occur heterogeneously within the particle because the drug solution cannot penetrate entirely into the very porous PLLA in the adsorption process. The resulted localization of the drug makes it difficult to control the drugrelease kinetics.

In this study, we further explored the possibility of using FDPLLA as a drug carrier by developing new PLLA/drug blend particles without using the adsorption method. We used rifampicin $\left(\mathrm{C}_{43} \mathrm{H}_{58} \mathrm{~N}_{4} \mathrm{O}_{12}\right.$, molar mass: 822.95 Da), an antibiotic drug used as an antibacterial and antifungal agent, as the drug compound. We considered rifampicin to be highly suitable for the present study because it is soluble in both 1,4dioxane and water; the former is a unique solvent that can be used to freeze-dry PLLA. In addition, rifampicin exhibits maximum absorbance at $473 \mathrm{~nm}$, which allowed us to easily measure the concentration of the drug that is released in 
water using UV-visible spectroscopy. First, we prepared a solution that was composed of PLLA, rifampicin, and 1,4dioxane; then, the solution was freeze-dried to obtain blend particles composed of PLLA and rifampicin. The obtained blend material has the following advantages over the BSAloaded FDPLLA developed in the previous study (simple adsorption method): (1) the blend material is macroscopically homogeneous such that the drug release is expected to be very stable; thus, reproducible release data can be obtained; (2) the amount of drug loading can be easily controlled by the solution content. We investigated the structure and release kinetics of blend materials prepared under different conditions. For comparison, we also executed the release experiments on the PLLA/rifampicin particles prepared by the simple adsorption method.

\section{Experimental}

The PLLA used in this study was supplied by Mitsui Chemicals, Tokyo, Japan. The polymer contained 98\% L units, and its molar mass was $210 \mathrm{kDa}$. Rifampicin was purchased from Tokyo Chemical Industry, Tokyo, Japan, and was used as received. Two solutions containing PLLA/rifampicin/1,4dioxane in ratios of 2.0/1.0/98 and 5.0/2.5/95 by weight were prepared. The PLLA content of the solution is denoted by $C_{\text {PLLA }}$; therefore, $C_{\text {PLLA }}=2.0 \mathrm{wt} \%$ for the former solution and $C_{\text {PLLA }}=4.9 \mathrm{wt} \%$ for the latter solution. The solutions were frozen using two methods: (a) pouring dropwise into liquid nitrogen (drop method) and (b) spraying the solution as a mist into liquid nitrogen (spray method). The frozen materials were kept under vacuum for $24 \mathrm{~h}$ to remove the solvent by sublimation, and additional vacuum drying was performed for $24-700 \mathrm{~h}$ at room temperature. The diameters of the obtained particles prepared using methods (a) and (b) were $2-3 \mathrm{~mm}$ and $10-200 \mu \mathrm{m}$, respectively. A typical appearance of the blend particles obtained by method (a) is shown in Figure 1. Drug release was executed in water $(\mathrm{pH}=7.0)$ at $37.0^{\circ} \mathrm{C}$. Typically, $4.5 \mathrm{mg}$ of the blend particles was immersed in $50 \mathrm{~mL}$ of water. Prior to release, water was permeated into the blend particles under reduced pressure. The procedure was described in detail in our previous paper [11]. This permeation procedure was required to obtain reproducible release data, because PLLA is highly hydrophobic.

The amount of rifampicin released in water was monitored by measuring the absorbance at $473 \mathrm{~nm}$, where the maximum absorbance of rifampicin in water is observed. The molar extinction coefficient at this wavelength was estimated to be $15,900 \mathrm{M}^{-1} \mathrm{~cm}^{-1}$. A Hitachi U-3900H UV spectrometer was used for monitoring, and release profiles of rifampicin with respect to time were obtained.

For comparison, we also prepared rifampicin-loaded particles by a simple adsorption method [11]: pure PLLA was freeze-dried from a 1,4-dioxane solution $\left(C_{\mathrm{PLLA}}=2.0\right.$, 5.0 , and $8.0 \mathrm{wt} \%)$ by the drop method. The average diameter of the obtained porous PLLA particles was $2.3 \mathrm{~mm}$, which was nearly independent of $C_{\text {PLLA }}$. The particles were immersed in a $0.20 \mathrm{wt} \%$ aqueous rifampicin solution by the permeation method under reduced pressure as was

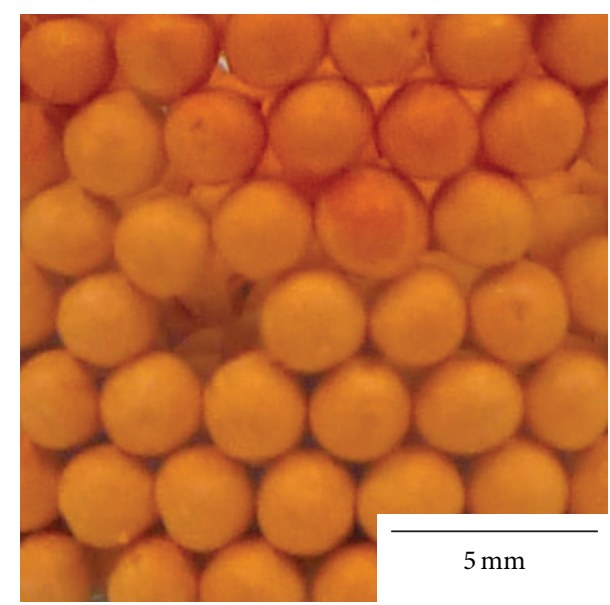

FIGURE 1: PLLA/rifampicin blend particles prepared by the drop freeze-drying method with $C_{\text {PLLA }}=4.9 \mathrm{wt} \%$.

performed at the beginning of the release experiment (see above). The particles were dried under vacuum for $24 \mathrm{~h}$. The rifampicin contents in the obtained particles were 6.2, 5.6, and $0.4 \mathrm{wt} \%$ for $C_{\text {PLLA }}=2.0,5.0$, and $8.0 \mathrm{wt} \%$, respectively. These rifampicin contents are lower than those obtained for the blend particles (33.3 wt\%), which is partially due to the low concentration of the rifampicin aqueous solution; a higher concentration could not be achieved because of the low solubility of rifampicin. Drug-release experiments were performed using these rifampicin-adsorbed PLLAs under the same conditions used to test the blend materials.

The morphology of the particles was examined using a Hitachi S-2600H scanning electron microscope (SEM). In order to observe the interior of the particles, the surface part was peeled away using adhesive tape. The specific surface area $\sigma$ was evaluated by the Brunauer-Emmett-Teller (BET) adsorption isotherm of krypton. The volume fraction of voids (void fraction) $r_{v}$ for the larger particles (drop method) was evaluated from the mass and apparent size of the particles. For the density of amorphous PLLA, we used the value of $1.248 \mathrm{~g} \mathrm{~cm}^{-3}$ reported in the literature [17]. Wide-angle Xray scattering (WAXS) measurements were performed using a Rigaku RINT2100 diffractometer with a $\mathrm{CuK} \alpha$ radiation source $(0.154 \mathrm{~nm})$.

\section{Results and Discussion}

3.1. Morphology. The obtained PLLA/rifampicin blend particles exhibited a high porosity similar to that obtained by the freeze-dried particles of pure PLLA [15]. Figure 2 shows SEM micrographs of the larger particles that were prepared by the drop method. For the particles with $C_{\text {PLLA }}=2.0 \mathrm{wt} \%$, both the surface and interior were observed to be highly porous. On the other hand, the surfaces of the particles with $C_{\text {PLLA }}=$ $4.9 \mathrm{wt} \%$ were not highly porous, exhibiting only very small holes; however, their interiors were still highly porous. The porous morphology observed for the particle interiors was composed of fine membranes, the thickness of which was 


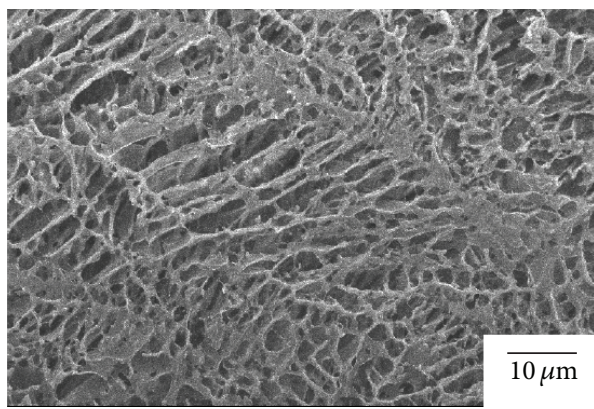

(a)

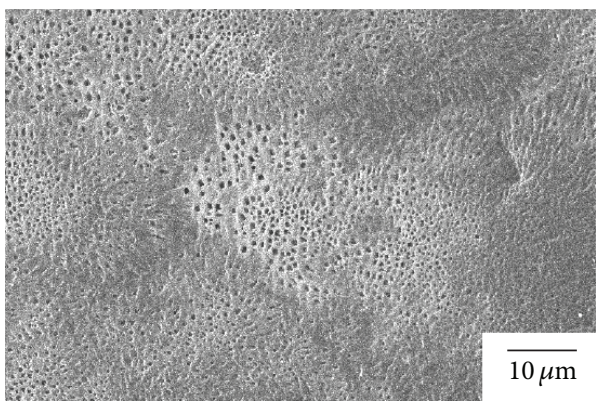

(c)

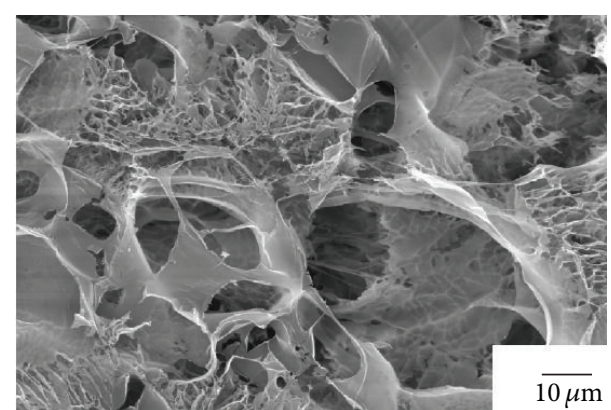

(b)

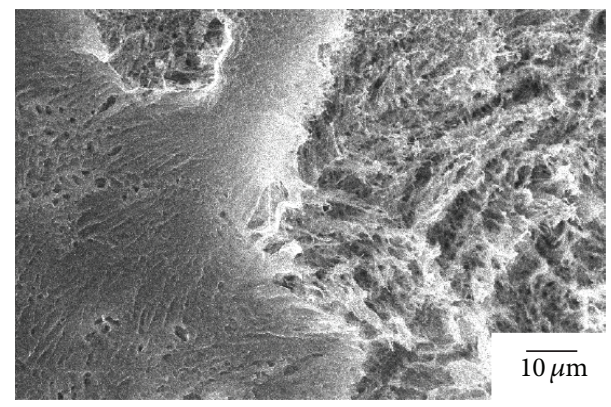

(d)

FIGURE 2: SEM images of the surface and interior of PLLA/rifampicin blend particles prepared by the drop freeze-drying method. (a) $C_{\text {PLLA }}=$ $2.0 \mathrm{wt} \%$, surface, (b) $C_{\mathrm{PLLA}}=2.0 \mathrm{wt} \%$, interior, (c) $C_{\mathrm{PLLA}}=4.9 \mathrm{wt} \%$, surface, and (d) $C_{\mathrm{PLLA}}=4.9 \mathrm{wt} \%$, interior.

TABLE 1: Morphological parameters of the freeze-dried PLLA/rifampicin particles.

\begin{tabular}{lccccc}
\hline$C_{\text {PLLA }} /$ wt $\%$ & Method & $d / \mathrm{mm}$ & $r_{v}$ & $\sigma / \mathrm{m}^{2} \mathrm{~g}^{-1}$ & $A_{v} / A_{t}$ \\
\hline 2.0 & Drop & 2.4 & 0.956 & 19.6 & 0.58 \\
2.0 & Spray & $0.010-0.10$ & & 34.8 & 0.85 \\
4.9 & Drop & 2.6 & 0.907 & 15.9 & 0.61 \\
4.9 & Spray & $0.010-0.20$ & & 33.7 & 0.022 \\
\hline
\end{tabular}

roughly estimated from the specific surface area $\sigma$ to be $24-$ $52 \mathrm{~nm}$ (the thickness of a thin film that has the same $\sigma$ value as that of the sample). Figure 3 shows SEM images of the smaller particles that were prepared by the spray method. The results indicate the same trend observed for the particles created by the drop method.

The morphological parameters of the particles are listed in Table 1 . The average particle diameter $d$ depends strongly on the freezing method, as mentioned in the experimental section. The void fraction $r_{v}$ is greater than $90 \%$, which indicates that the particles are highly porous even with a high content of the drug (ca. 33\%). The specific surface area $\sigma$ for the smaller particles is approximately twice as large as that for the larger particles. This discrepancy may be understood simply by considering the contour size effect of the particles. In addition, the difference in the rate of freezing may be responsible for this finding: during the freezing process, the phase separation of the solutes from the solvent occurs as the solvent crystallizes; however, for smaller droplets, freezing occurs more rapidly due to faster thermal conduction, which prohibits the formation of large domains of solutes and thus results in a finer microporous morphology. We also note that $\sigma$ decreases with increasing $C_{\text {PLLA }}$, which suggests that the phase separation of solutes and solvent occurs more slowly for higher values of $C_{\text {PLLA }}$.

Surface porosity $A_{v} / A_{t}$ was evaluated from the SEM images, and the results are shown in Table 1 , where $A_{v}$ is the void area on the surface of a particle and $A_{t}$ is the total surface area. The surface porosity for $C_{\text {PLLA }}=4.9 \mathrm{wt} \%$ is surprisingly lower than that for $C_{\text {PLLA }}=2.0 \mathrm{wt} \%$. Considering the fact that the $\sigma$ values are still high even for $C_{\mathrm{PLLA}}=4.9 \mathrm{wt} \%$, the low porosity must be limited to the surface region. Thus, we infer that a low-porosity skin exists on the surface of the higher- $C_{\text {PLLA }}$ particles. Such a skin may be formed during the freezing process in liquid nitrogen: when the solution is frozen, the solvent crystallizes excluding the solutes (PLLA and rifampicin), and as a result, a solution phase with a higher concentration of the solutes is formed, which leads to a dense skin near the surface region. For a lower- $C_{\text {PLLA }}$ solution, the amount of the solutes is too small to form a dense skin.

Figure 4 shows the WAXS profiles for the as-received rifampicin and the PLLA/rifampicin blend particles. The asreceived rifampicin was in a crystalline state as evidenced by a number of diffraction peaks (curve (a)), but once it 


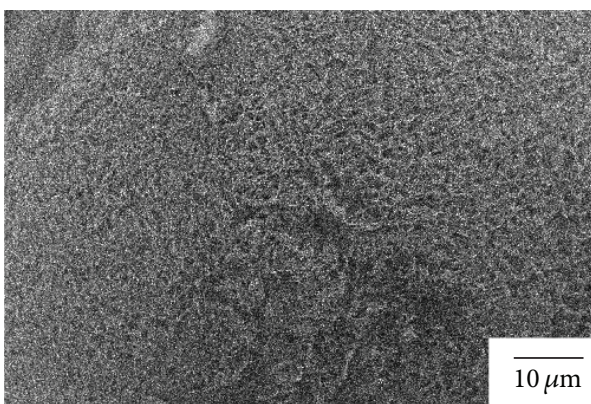

(a)

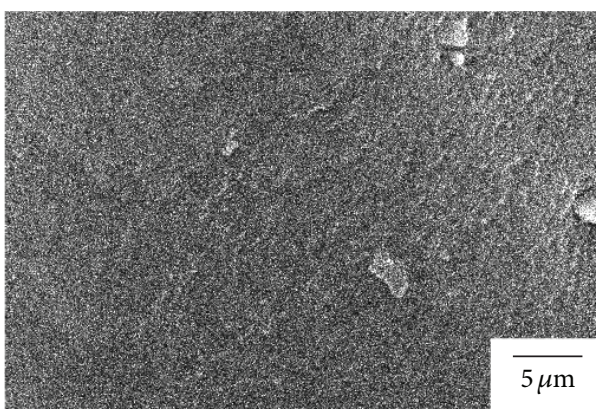

(c)

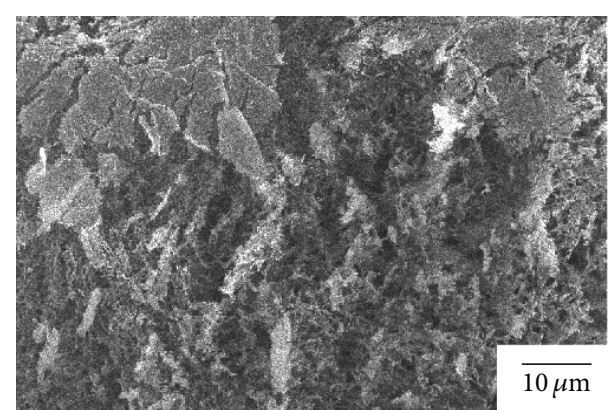

(b)

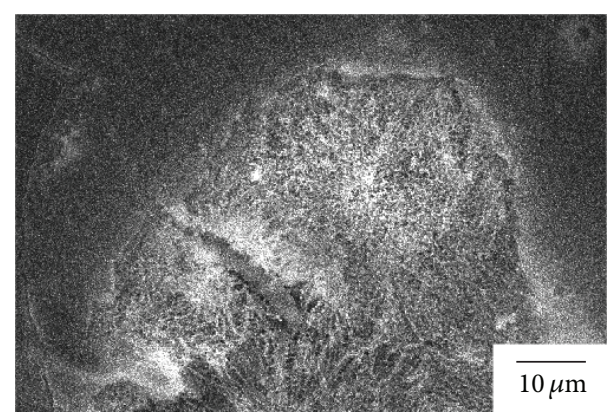

(d)

FIGURE 3: SEM images of the surface and interior of PLLA/rifampicin blend particles prepared by the spray freeze-drying method. (a) $C_{\text {PLLA }}=$ $2.0 \mathrm{wt} \%$, surface, (b) $C_{\text {PLLA }}=2.0 \mathrm{wt} \%$, interior, (c) $C_{\text {PLLA }}=4.9 \mathrm{wt} \%$, surface, and (d) $C_{\text {PLLA }}=4.9 \mathrm{wt} \%$, interior.

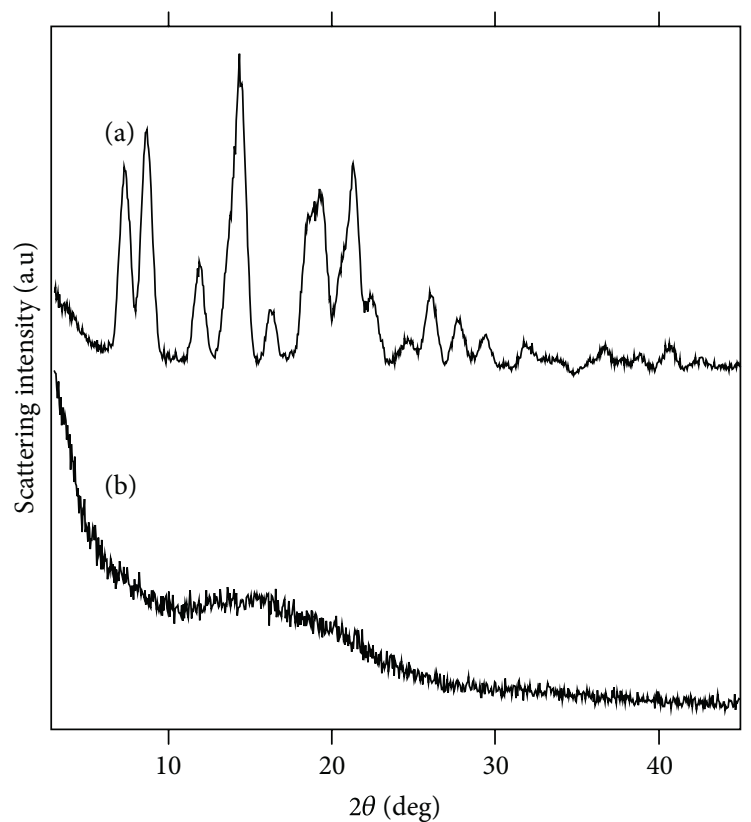

FIGURE 4: WAXS intensity profiles for (a) as-received rifampicin, and (b) PLLA/rifampicin blend particle prepared by the drop method from the $C_{\text {PLLA }}=4.9$ wt $\%$ solution. The wavelength of the $\mathrm{X}$-rays was $0.154 \mathrm{~nm}$. The vertical axis is appropriately shifted.

was freeze-dried together with PLLA, the rifampicin became completely amorphous (curve (b)). This result suggests that the compatibility of PLLA with rifampicin is rather high such that PLLA prevents the formation of rifampicin domains that are large enough to allow for crystallization. Figure 4(b) indicates that the PLLA in the blend particles was also in an amorphous state. The same result has already been confirmed for pure freeze-dried PLLAs.

3.2. Release Kinetics. Figure 5 shows the time evolution of rifampicin release in water for the PLLA/rifampicin blend particles. The curves indicate that the degree of release $M(t) / M_{\infty}$ reached an ultimate value within $24 \mathrm{~h}$. The total amount of the drug released in water after $24 \mathrm{~h}$ was estimated to be $70-89 \%$ of that initially existing in the PLLA particles; the values of the final release ratio $R_{\infty}=M_{\infty} / M_{\text {fed }}$ are shown in Table 1 , where $M_{\text {fed }}$ is the mass of rifampicin that initially existed in the PLLA/rifampicin particle. The results indicate that there existed a fraction of rifampicin that could not be extracted in the release experiment, because the drug was completely surrounded by PLLA in this case. Considering the mass ratio of rifampicin/PLLA $(=1.0 / 2.0)$ for the present blend particles, it is likely that PLLA exists as a continuous phase. Because of the hydrophobicity of PLLA, water hardly penetrates into the PLLA phase, which consequently prevents the fraction of the drug surrounded by the PLLA phase from being released as long as PLLA remains intact.

It was observed that the time required to reach the ultimate value of $M(t)$ is generally shorter for low values of $C_{\text {PLLA }}$. The result may be due to the higher porosity of the lower- $C_{\text {PLLA }}$ samples, as revealed by the values of $r_{v}$ and $A_{v} / A_{t}$ in Table 1. Assuming that the interior pore space of the particles during release is filled with an aqueous phase 


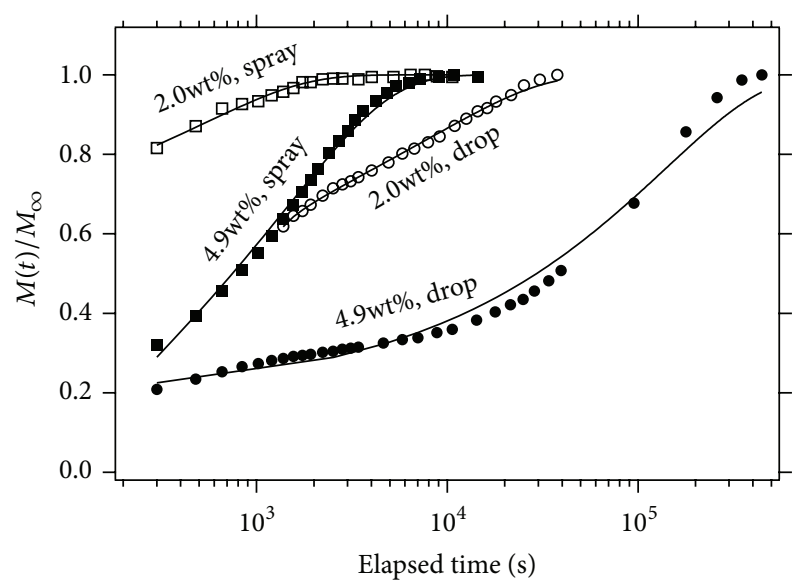

Figure 5: Time evolution of drug release $M(t) / M_{\infty}$ from the PLLA/rifampicin blend particles in water at $37.0^{\circ} \mathrm{C} . C_{\text {PLLA }}$ and method of preparation are indicated in the figure. The solid curves indicate the results of the fitting analysis.

and that this phase provides a diffusion path for the drug, the larger $r_{v}$ promotes drug transport. It is also noted that the time required to reach the ultimate value of $M(t)$ is longer for larger particles. For the smaller particles, the diffusion path in the interior of the particle is shorter, which makes the completion of release occur more rapidly.

In this section, we analyze the time-evolution data of release. Assuming a Fickian diffusion model for a nonswellable sphere, $M(t) / M_{\infty}$ is expressed as [18]

$\frac{M(t)}{M_{\infty}}=(1-b)\left\{1-\frac{6}{\pi^{2}} \sum_{n=1}^{\infty} \frac{1}{n^{2}} \exp \left[-\frac{D n^{2} \pi^{2}\left(t-t_{i}\right)}{a^{2}}\right]\right\}+b$,

where $D$ is the diffusion coefficient of the drug in the particle, $a$ is the radius of the particle $(d / 2), b$ is a baseline parameter that accounts for very rapid release at an early stage (initial burst), and $t_{i}$ is the induction period for the second step of the release after the initial burst. We performed nonlinear least-squares fitting analysis in which $D, b$, and $t_{i}$ were treated as variable parameters. Note that the obtained $D$ values do not include a contribution from the initial burst and that the analysis is based on the assumption that the interior of the particle is regarded as a homogeneous medium. The latter assumption implies that the estimated $D$ actually denotes the average diffusion coefficient over the PLLA/rifampicin solid phase and aqueous phase in the particle.

The results of the analysis are shown in Table 2. The apparent diffusion coefficient $D$ is greater for the particles with $C_{\mathrm{PLLA}}=2.0 \mathrm{wt} \%$ than those with $C_{\mathrm{PLLA}}=4.9 \mathrm{wt} \%$. This difference may be due to the higher surface porosity of the former samples, as evidenced by the $A_{v} / A_{t}$ values. It is also noted that $D$ is smaller for the smaller particles. For the smaller particles, the penetration of the aqueous phase into the particle interior may be incomplete because of the very fine structure of the hydrophobic PLLA surface, which results in the suppression of drug diffusion.

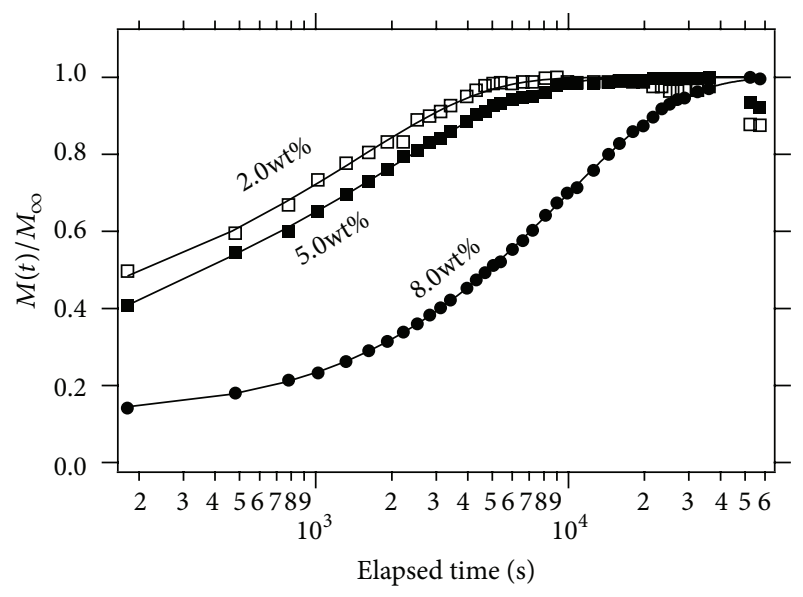

FIgURE 6: Time evolution of drug release $M(t) / M_{\infty}$ in water at $37.0^{\circ} \mathrm{C}$ from the rifampicin-adsorbed FDPLLAs. The values of $C_{\mathrm{PLLA}}$ are indicated in the figure. The solid curves indicate the results of the fitting analysis.

TABLE 2: Best-fit parameters obtained from the analysis of release profiles.

\begin{tabular}{lcccc}
\hline$C_{\mathrm{PLLA}} / \mathrm{wt} \%$ & Method & $b$ & $t_{i} / 10^{2} \mathrm{~s}$ & $D / \mathrm{m}^{2} \mathrm{~s}^{-1}$ \\
\hline 2.0 & Drop & 0.55 & 12.5 & $1.2 \times 10^{-11}$ \\
2.0 & Spray & 0.64 & 0.4 & $8.7 \times 10^{-14}$ \\
4.9 & Drop & 0.16 & 0 & $8.9 \times 10^{-13}$ \\
4.9 & Spray & 0 & 1.4 & $2.6 \times 10^{-14}$ \\
2.0 & Adsorption & 0.26 & 0 & $2.2 \times 10^{-10}$ \\
5.0 & Adsorption & 0.31 & 0 & $1.4 \times 10^{-10}$ \\
8.0 & Adsorption & 0 & 0 & $3.8 \times 10^{-11}$ \\
\hline
\end{tabular}

We observed an initial burst of release for the blend systems studied. The baseline parameter $b$ is a measure of the initial burst. It is likely that the initial burst reflects the rapid release from the region near the surface of the particles. The initial burst observed was less prominent for the samples with $C_{\mathrm{PLLA}}=4.9 \mathrm{wt} \%$ than for those with $C_{\mathrm{PLLA}}=2.0 \mathrm{wt} \%$. This difference may be related to the high surface porosity of the former, that is, release from the near-surface region requires only a very short path for the drug to diffuse, but the existence of the denser skin at the surface still suppresses the diffusion for the higher- $C_{\text {PLLA }}$ particles.

Figure 6 shows the release profiles for the rifampicinadsorbed PLLAs; the corresponding best-fit parameters are shown in Table 2 . The results show that the time required to reach the ultimate value of $M(t)$ lies between that of the blend sample created using the spray method and that of the sample created using the drop method. On the other hand, the diffusion coefficient $D$ is greater than that for the blend samples. It is reasonable to consider that the rifampicin simply adsorbed to the surface of FDPLLA can be easily desorbed, which leads to an apparently higher $D$ value. We also observed that the final release ratio $R_{\infty}=M_{\infty} / M_{\text {fed }}$ reaches nearly $100 \%$. This finding suggests that there is no fraction of rifampicin that is completely surrounded by the PLLA phase. For the sample with $C_{\text {PLLA }}=8.0 \mathrm{wt} \%$, an initial 
burst was not observed (Table 2), likely due to the very low amount of rifampicin loaded ( $0.4 \mathrm{wt} \%$ with respect to PLLA) compared with the amount loaded in the other two samples (6.2 and $5.6 \mathrm{wt} \%$ ), as mentioned in the experimental section. Indeed, the porosity of the FDPLLA particles with $C_{\text {PLLA }}=$ $8.0 \mathrm{wt} \%$ is extremely low $\left(\sigma=8.4 \mathrm{~m}^{2} \mathrm{~g}^{-1}\right.$, and $\left.A_{v} / A_{t}=3.0 \%\right)$, and this low porosity may be responsible for the low drug loading.

\section{Conclusions}

We observed that the present PLLA/rifampicin blend particles exhibit effective drug release into an aqueous phase. The release rate significantly depends on the morphology of the particles, specifically the particle size, specific surface area, and surface porosity. We confirmed that the morphology can be modified by the concentration $C_{\text {PLLA }}$ and by the method of freezing; in particular, the latter can control the size of the resulting particles. Furthermore, the amount of drug loaded into the blend particles was observed to be prominently higher than that loaded into the rifampicin-adsorbed PLLAs; thus, the present blend system is a promising candidate as a drug carrier. The final release ratio $R_{\infty}$ did not reach $100 \%$ over the time range investigated, but the remaining fraction of the drug could be released after the degradation of PLLA. Controlling the phase-separated structure of rifampicin and PLLA in the blend to obtain the desired fraction of the drug surrounded completely by PLLA would allow us to achieve drug delivery over a longer time span, where the release is dominated by the decomposition of the PLLA phase.

\section{Conflict of Interests}

The authors declare that there is no conflict of interests regarding the publication of this paper.

\section{References}

[1] S. Freiberg and X. X. Zhu, "Polymer microspheres for controlled drug release," International Journal of Pharmaceutics, vol. 282, no. 1-2, pp. 1-18, 2004.

[2] D. Lensen, K. van Breukelen, D. M. Vriezema, and J. C. M. van Hest, "Preparation of biodegradable liquid core PLLA microcapsules and hollow PLLA microcapsules using microfluidics," Macromolecular Bioscience, vol. 10, no. 5, pp. 475-480, 2010.

[3] A. El Fagui and C. Amiel, "PLA nanoparticles coated with a beta-cyclodextrin polymer shell: preparation, characterization and release kinetics of a hydrophobic compound," International Journal of Pharmaceutics, vol. 436, pp. 644-651, 2012.

[4] S. Zhou, X. Deng, M. Yuan, and X. Li, "Investigation on preparation and protein release of biodegradable polymer microspheres as drug-delivery system," Journal of Applied Polymer Science, vol. 84, no. 4, pp. 778-784, 2002.

[5] U. Paiphansiri, P. Tangboriboonrat, and K. Landfester, "Polymeric nanocapsules containing an antiseptic agent obtained by controlled nanoprecipitation onto water-in-oil miniemulsion droplets," Macromolecular Bioscience, vol. 6, no. 1, pp. 33-40, 2006.
[6] S. Mao, C. Guo, Y. Shi, and L. C. Li, "Recent advances in polymeric microspheres for parenteral drug delivery. Part 1," Expert Opinion Drug Delivery, vol. 9, pp. 1161-1176, 2012.

[7] S. Mao, C. Guo, Y. Shi, and L. C. Li, "Recent advances in polymeric microspheres for parenteral drug delivery. Part 2," Expert Opinion Drug Delivery, vol. 9, pp. 1209-1223, 2012.

[8] R. T. Liggins and H. M. Burt, "Paclitaxel loaded poly(Llactic acid) microspheres: properties of microspheres made with low molecular weight polymers," International Journal of Pharmaceutics, vol. 222, no. 1, pp. 19-33, 2001.

[9] R. T. Liggins and H. M. Burt, "Paclitaxel loaded poly(L-lactic acid) (PLLA) microspheres. II. The effect of processing parameters on microsphere morphology and drug release kinetics," International Journal of Pharmaceutics, vol. 281, no. 1-2, pp. 103106, 2004.

[10] Z. Wang, "Effects of the process parameters on the initial burst release of poly(lactide-co-glycolide) microspheres containing bovine serum albumin by the double-emulsion solvent evaporation/extraction method," Journal of Applied Polymer Science, vol. 115, no. 5, pp. 2599-2608, 2010.

[11] T. Sasaki, K. Tanaka, D. Morino, and K. Sakurai, "Morphology and release kinetics of protein-loaded porous poly(L-lactic acid) spheres prepared by freeze-drying technique," ISRN Pharmaceutics, vol. 2011, Article ID 490567, 8 pages, 2011.

[12] A. Bohr, M. Yang, S. Baldursdottir et al., "Particle formation and characteristics of Celecoxib-loaded poly(lactic-co-glycolic acid) microparticles prepared in different solvents using electrospraying," Polymer, vol. 53, pp. 3220-3229, 2012.

[13] Y. Baimark and M. Srisa-Ard, "Preparation of drug-loaded microspheres of linear and star-shaped poly(D,L-lactide)s and their drug release behaviors," Journal of Applied Polymer Science, vol. 124, no. 5, pp. 3871-3878, 2012.

[14] W. Zheng and S. L. Simon, "Polystyrene freeze-dried from dilute solution: Tg depression and residual solvent effects," Polymer, vol. 47 , no. 10, pp. 3520-3527, 2006.

[15] T. Sasaki, D. Morino, and N. Tabata, "Origin of enhanced cold crystallization rate for freeze-dried poly(L-lactide) from solutions," Polymer Engineering and Science, vol. 51, no. 9, pp. 1858-1865, 2011.

[16] T. Sasaki, N. Yamauchi, S. Irie, and K. Sakurai, "Differential scanning calorimetry study on thermal behaviors of freezedried poly(L-lactide) from dilute solutions," Journal of Polymer Science B, vol. 43, no. 2, pp. 115-124, 2005.

[17] E. W. Fischer, H. J. Sterzel, and G. Wegner, "Investigation of the structure of solution grown crystals of lactide copolymers by means of chemical reactions," Kolloid-Zeitschrift und Zeitschrift für Polymere, vol. 251, no. 11, pp. 980-990, 1973.

[18] P. L. Ritger and N. A. Peppas, "A simple equation for desciption of solute release I. Fickian and non-Fickian release from nonswellable devices in the form of slabs, spheres, cylinders or discs," Journal of Controlled Release, vol. 5, no. 1, pp. 23-36, 1987. 

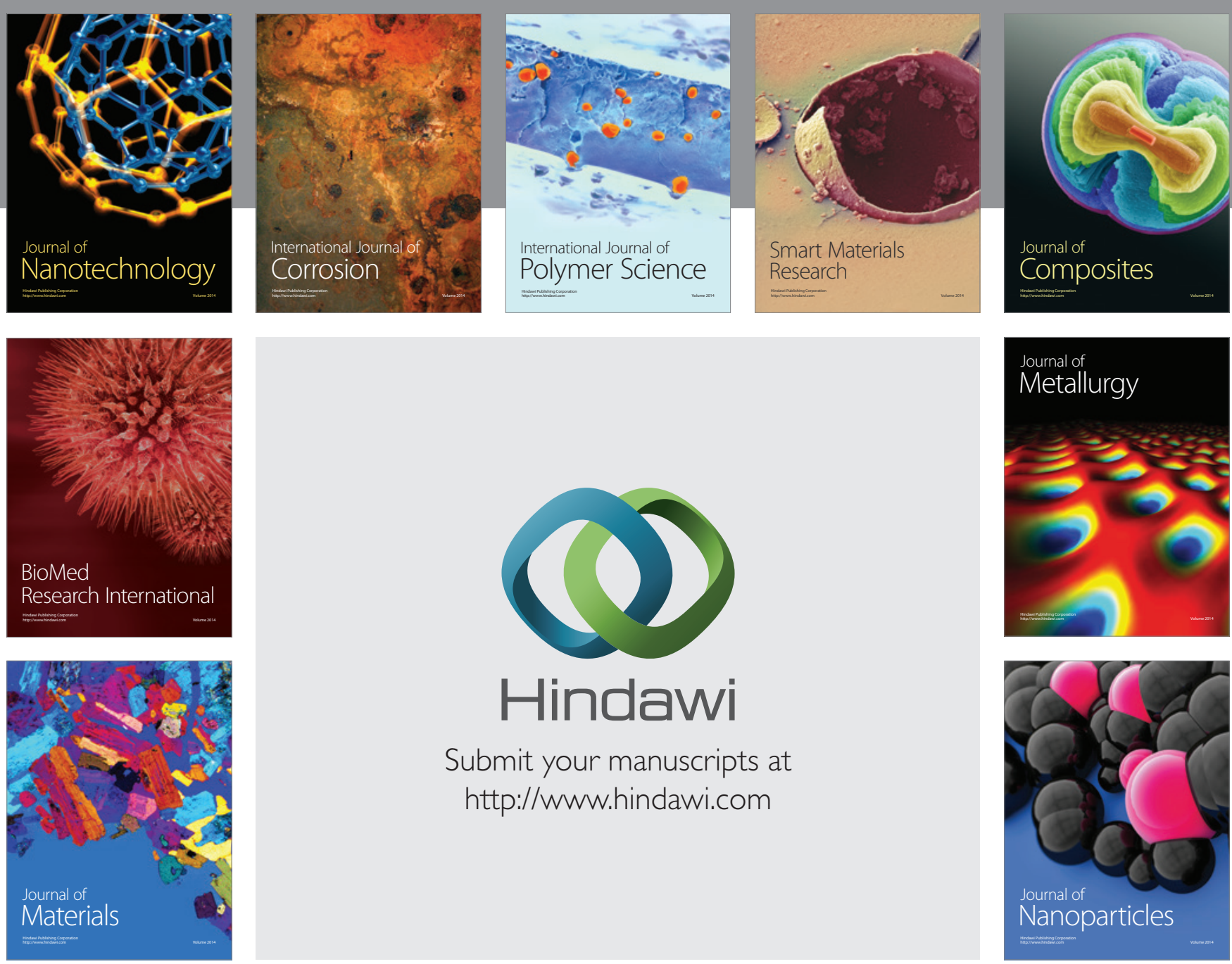

Submit your manuscripts at http://www.hindawi.com
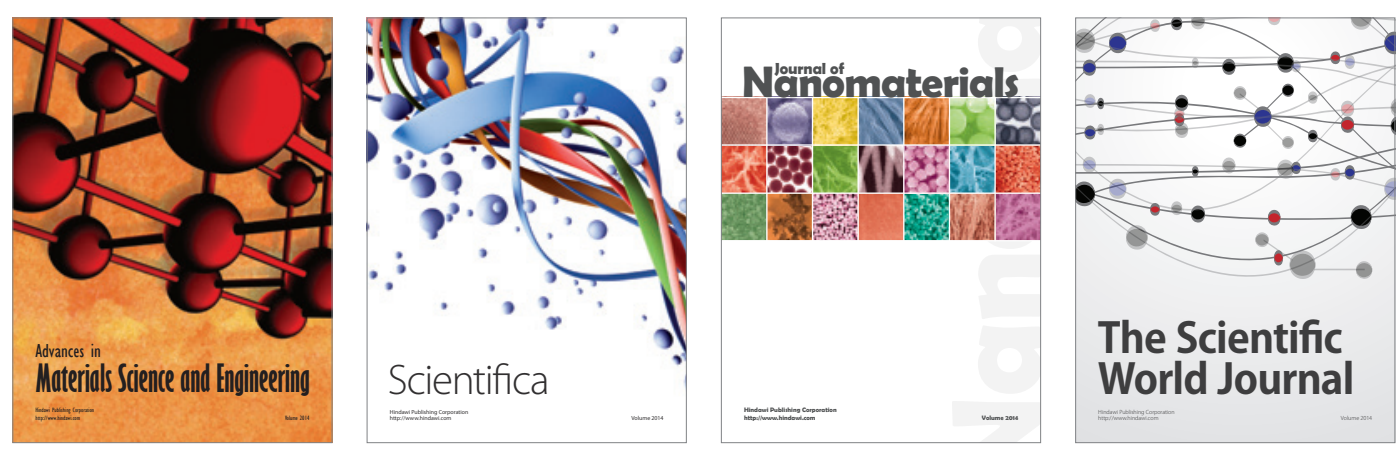

\section{The Scientific World Journal}
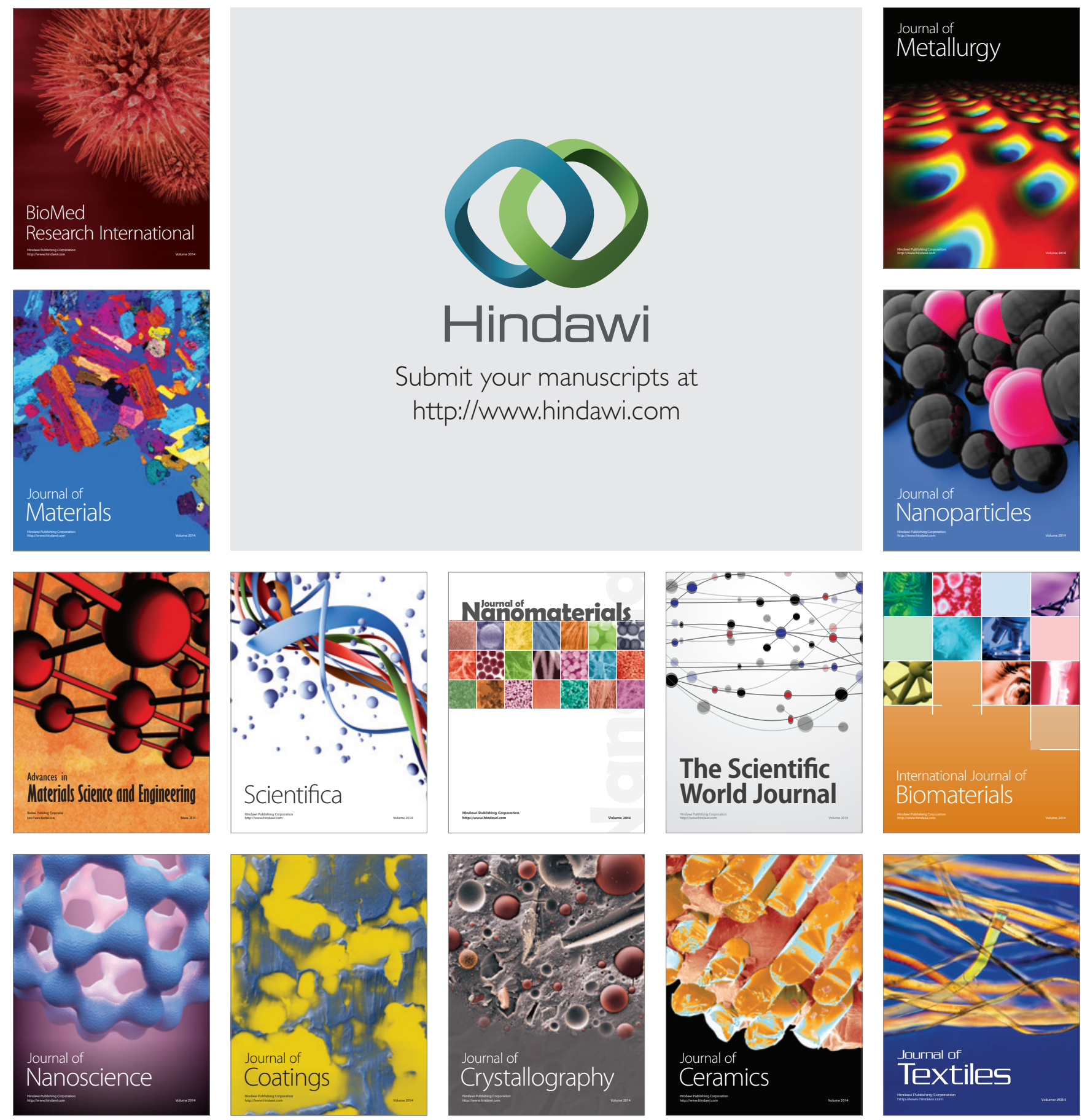